\title{
Chaotic Temperature Dependence at Zero Temperature
}

\author{
A. C. D. van Enter ${ }^{1,2}$ and W. M. Ruszel ${ }^{1,2}$
}

Received September 8, 2006; accepted November 14, 2006

Published Online: January 30, 2007

\begin{abstract}
We present a class of examples of nearest-neighbour, bounded-spin models, in which the low-temperature Gibbs measures do not converge as the temperature is lowered to zero, in any dimension.
\end{abstract}

KEY WORDS: Chaotic Temperature Dependence, vector spin models, zerotemperature limits of Gibbs states, non-existence

\section{INTRODUCTION}

In most examples we know in statistical mechanics, ground states measures, or at least a subset of the set of ground states measures, can be obtained as proper limits of low-temperature states (Gibbs measures). In fact, the same tends to be true at positive temperatures: changing the temperature leads to a limit of Gibbs measures (in the weak $*$-topology) approaching some other Gibbs measure at the new temperature. It is not unususal that a limit of pure (extremal) Gibbs measures is non-pure, as often happens for low-dimensional ground states, and as also at positive temperatures is known for example in the "Thouless effect." ${ }^{(1,11)}$ Also examples are known where there exists an infinite sequence of first-order phase transitions at low temperatures, but still there is the possibility of convergence to at least some ground state as the temperature approaches zero, see e.g., Refs. 3, 9. In this note we present an example where there is no low-temperature limit of any sequence of Gibbs measures for a prescribed sequence of temperatures. Indeed,

\footnotetext{
${ }^{1}$ Center for Theoretical Physics, University of Groningen, Nijenborgh 4, 9747AG, Groningen, The Netherlands. e-mail: aenter@phys.rug.nl

${ }^{2}$ Department of Mathematics and Computing Science, University of Groningen, Blauwborgje 3, 9747AC, Groningen, The Netherlands.
} 
lowering the temperature will mean that one oscillates between Gibbs measures having either a ferromagnetic or antiferromagnetic character. Such a "messy" (10) behaviour seems an example of the "chaotic temperature dependence," which has been proposed in the spin-glass literature, see e.g., Refs. 7, 8. (We use here the terminology "chaotic" for non-convergence, in the same spirit as it was introduced in the notions of "chaotic size dependence" and "chaotic time dependence" by Newman and Stein. Unfortunately the term "chaotic" has been used before in the literature in the different, rather weaker sense of "chaotic behaviour under Renormalisation Group maps." ${ }^{(6)}$ This notion describes messy behaviour of longrange correlations, but not of short-range correlations as we find here. We thank A. Nihat Berker for a helpful discussion on this point.) Our example employs bounded spins with a bounded interaction. The spins take continuous values, and we don't know if similar behaviour can occur for discrete spins. Also, our interaction is not continuous, but this is not an essential feature, and the example could be made continuous. Although the notion of chaotic temperature dependence was introduced in the spin-glass literature, our interactions do not contain disorder, but are deterministic.

\section{NOTATION AND RESULT}

We will say that Chaotic Temperature Dependence (CTD) holds, if no ground state measure can be obtained as a proper limit of finite-temperature Gibbs measures $^{(4)}$ by lowering the temperatures. (This does allow convergence on certain subsequences of temperatures, as indeed necessarily happens due to compactness arguments).

We consider for simplicity 2-component spins (the generalization to $\mathrm{N}$ component spins is immediate), which live on the unit circle and are described by the angle $\theta$, and which are located on a $d$-dimensional lattice $\mathbb{Z}^{d}$, with the following (formal) Hamiltonian:

$$
H=-\sum_{\langle i, j\rangle \in \mathbb{Z}^{d}} U\left(\theta_{i}-\theta_{j}\right)
$$

The potential $U$ is a sum of two "Seuss" potentials having the form of wells-inwells (hats-in-hats), ${ }^{(3,12)}$ one ferromagnetic and the other one antiferromagnetic:

$$
\begin{aligned}
U(x)= & \sum_{n \geq 1}\left(\frac{1}{2^{2 n}}+\frac{1}{2^{2 n+1}}\right) \mathbb{1}_{\left[\frac{-\varepsilon_{2 n}}{2}, \frac{\varepsilon_{2 n}}{2}\right]}(x) \\
& +\sum_{n \geq 1}\left(\frac{1}{2^{2 n+1}}+\frac{1}{2^{2 n+2}}\right) \mathbb{1}_{\left[\frac{-\varepsilon_{2 n+1}}{2}, \frac{\varepsilon_{2 n+1}}{2}\right]}(\pi-x)+\frac{1}{4} \mathbb{1}_{\left[-\frac{\varepsilon_{1}}{2}, \frac{\varepsilon_{1}}{2}\right]}(\pi-x)
\end{aligned}
$$

with $\varepsilon_{n}=\varepsilon^{3^{n}}$ for $\varepsilon$ small enough. The steps (wells) in the two sums have asymmetric widths ( $\varepsilon_{2 n}$ resp. $\left.\varepsilon_{2 n+1}\right)$ and heights (depths) but the maximum of both 


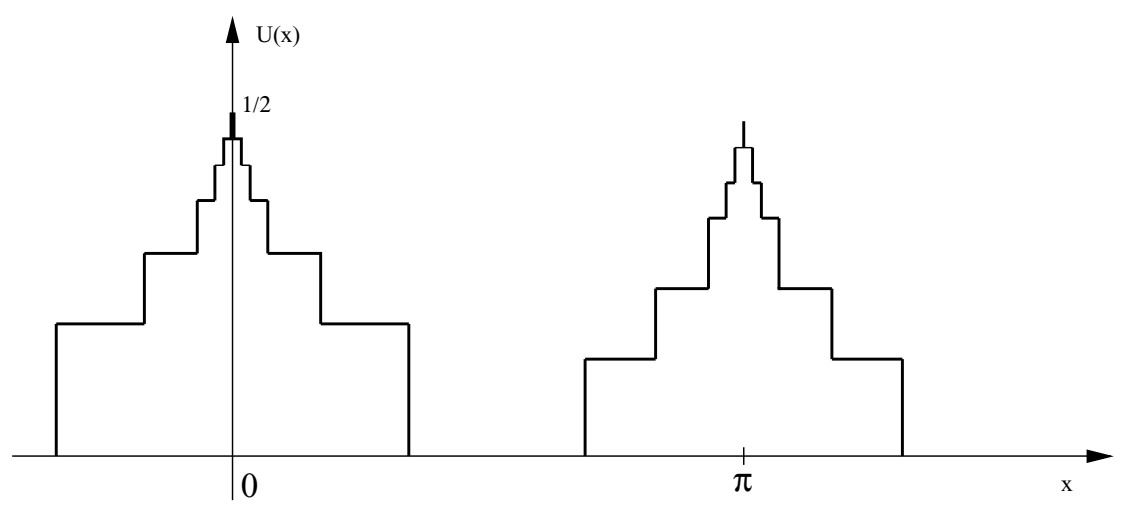

Fig. 1. Sum of two Seuss potentials.

terms (the two sums of the depths of the wells) is $\frac{1}{2}$. The first sum of the indicator functions contains the ferromagnetic terms, the second sum antiferromagnetic ones.

For an impression of our construction see Fig. 1 (however, with a large $\varepsilon$ ).

The construction is such that, precisely as in Ref. 3, there is, in $d$ at least 2, an infinite sequence of first-order transitions to ever deeper and narrower wells. However, now the successive wells alternate between being ferromagnetic and antiferromagnetic.

Lowering the temperature $T$ for this potential means that the typical bond configurations jump between successive wells centered at 0 and $\pi$, i.e., between a ferromagnetic resp. antiferromagnetic state.

Consider the set of Gibbs measures for the system with Hamiltonian $H$ at inverse temperature $\beta, \mathcal{G}_{\beta}(H)=\left\{\mu_{\beta}^{\alpha} \mid \alpha \in I\right\} . I$ is some index set and $\alpha$ labels the Gibbs measures in this set. We can therefore construct a sequence of temperatures $T_{n}$ (or equivalently inverse temperatures $\beta_{n}$ ), such that for any choice of $\alpha$ the limit $\mu_{\beta_{n}}^{\alpha}$ does not exist as $T_{n}$ goes to 0 , that is $\beta_{n}$ goes to $\infty$.

Theorem 2.1 Let $U$ be defined as in (3). For $N$-vector models in any dimension and $N \geq 2$, Chaotic Temperature Dependence (CTD) occurs at $T=0$.

Proof: We treat the cases $d \geq 2$ and $d=1$ separately.

a) For $d$ at least 2, the proof is an immediate corollary of Ref. 3. Indeed, take a sequence of temperatures such that the typical bonds for (any) choice of Gibbs measure are oscillating between ferromagnetic and antiferromagnetic, corresponding to the even-numbered and odd-numbered wells in Ref. 3. Then there are (at least) two limit points, a ferromagnetic and an 
antiferromagnetic one. In $d$ at most 2, presumably there will be only two (the rotation invariant mixtures of the pure configurations), in $d$ at least 3 , magnetized and Néel ordered ground state configurations are possible subsequence limits of various low-temperature Gibbs measures.

However, the phenomenon does not need the existence of these phase transitions and indeed occurs already in one dimension.

$b$ ) For the proof in $d=1$ we present an elementary argument. We look at the bond distributions at different bonds. Each bond independently takes values on the circle, describing the difference in spin values between neighboring sites. The bonds are thus i.i.d. on the circle $[0,2 \pi)$, so it suffices to look at the probability $P_{n}(\beta)$ of one bond being in precisely the $n$-th well at inverse temperature $\beta$ and to show that for appropriately chosen $\beta_{n}$ and $n$ this probability is larger than one half. Then either the probability that the system is in the ferromagnetic state (most bonds ferromagnetic) or the probability that it is in the antiferromagnetic state (most bonds antiferromagnetic) is larger than one half and the system keeps oscillating between those two if $\beta$ increases.

The probability that the bond is in the $n$-th well and not in the $n+2$-th well (by construction it is not in the $n+1$-th well) is

$$
P_{n}(\beta)=\frac{1}{Z_{\beta}}\left(\varepsilon_{n}-\varepsilon_{n+2}\right) \exp \left(-\frac{3 \beta}{2^{n+1}}\right)
$$

where $Z_{\beta}$ is a normalizing constant depending on the inverse temperature $\beta$. The probability $P_{n}(\beta)$, considered as a function of $n$, is maximal iff its logarithm (equals minus the $n$-th well's "free energy")

$$
-f_{\beta}(n):=-\frac{3 \beta}{2^{n+1}}+\ln \left(\varepsilon_{n}-\varepsilon_{n+2}\right)
$$

is maximal. As usual, maximal probability corresponds to minimal free energy. The derivative of $f$ with respect to $n$ (now taken as a continuous variable) is equal to

$$
f_{\beta}^{\prime}(n)=c_{1} \frac{\beta}{2^{n}}-c_{2}(\varepsilon) 3^{n}
$$

with $c_{i}$ strictly positive constants, $c_{2}$ depending on $\varepsilon$. Then it follows immediately that $f$ has its minimal value at $n_{\max }$ which satisfies

$$
6^{n_{\max }}=\frac{c_{1}}{c_{2}(\varepsilon)} \beta .
$$

It is clear that increasing $\beta$ means increasing $n_{\max }$ to get the most probable position of the bond. Choosing a sequence of inverse temperatures $\beta_{n}=O\left(6^{n}\right)$ and $\varepsilon$ appropriately (such that $n_{\max }=n$ is an integer) we get that the position of 
the most probable well $n_{\max }$ is alternating between even and odd, i.e., the bond concentrates itself alternatingly either at 0 or $\pi$.

Remark. If the minimum of $f$ is at a value between integers, there can be a two-fold degeneracy, with wells $n$ and $n+1$ being equally probable.

It remains to prove that $P_{n_{\max }}\left(\beta_{n}\right) \geq \frac{1}{2}+\delta$ for some small number $\delta$, for all suffiently large $n$, which is equivalent to

$$
P_{n_{\max }}\left(\beta_{n}\right) \geq \sum_{n \neq n_{\max }} P_{n}\left(\beta_{n}\right)+\delta
$$

or

$$
\left(\varepsilon_{n_{\max }}-\varepsilon_{n_{\max }+2}\right) \exp \left(-\frac{3 \beta_{n}}{2^{n+1}}\right) \geq \sum_{n \neq n_{\max }}\left(\varepsilon_{n}-\varepsilon_{n+2}\right) \exp \left(-\frac{3 \beta_{n}}{2^{n+1}}\right)+\delta .
$$

We prove the somewhat stronger result that the probability distribution over the wells becomes more and more sharply peaked; indeed the probability of $n$ being the favourite well increases to one when $n$ and the corresponding inverse temperature $\beta_{n}$ increase to infinity.

For the estimate we first neglect that the wells lie inside each other. Once $\varepsilon$ is chosen small enough, the mistake we make this way is sufficiently small that the inequality we will derive remains true.

Assume thus that the wells are separate, and thus the probability of being in the $n$-th well is

$$
\begin{aligned}
\tilde{P}_{n}(\beta) & =\frac{1}{\tilde{Z}_{\beta}} \exp \left(-\frac{3 \beta}{2^{n+1}}-c_{\varepsilon} 3^{n}\right) \\
& =\frac{1}{\tilde{Z}_{\beta}} \exp \left(-\tilde{f}_{\beta}(n)\right)
\end{aligned}
$$

where $\tilde{Z}_{\beta}$ is again a normalizing constant and $c_{\varepsilon}:=-\log (\varepsilon)$.

Using the same argument as before we get that the most probable position of the bond is at $n_{\max }$ which satisfies

$$
\beta=c_{\varepsilon} \frac{2}{3} \frac{\log (3)}{\log (2)} 6^{n_{\max }},
$$

i.e., at the minimal point of the function $\tilde{f}_{\beta}(n)$. (Here again we first consider the variable $n$ to be continuous and then look at integer values for the maximal $n$, choosing the sequence $\beta_{n}$ appropriately.) Then for $k \in \mathbb{N}$ note that

$$
\tilde{f}_{\beta_{n}}\left(n_{\max }+k\right)=3^{n_{\max }} c_{\varepsilon}\left(2^{-k} a+3^{k}\right)
$$


resp.

$$
\tilde{f}_{\beta_{n}}\left(n_{\max }-k\right)=3^{n_{\max }} c_{\varepsilon}\left(2^{k} a+3^{-k}\right)
$$

with the abbreviation $a:=\frac{\log (3)}{\log (2)}$.

Estimating the ratio $\frac{\tilde{P}_{n_{\max } \pm k}\left(\beta_{n}\right)}{\tilde{P}_{n_{\max }}\left(\beta_{n}\right)}$ we can show easily that

$$
\frac{\tilde{P}_{n_{\max } \pm k}\left(\beta_{n}\right)}{\tilde{P}_{n_{\max }}\left(\beta_{n}\right)} \leq \exp \left(-c_{\beta_{n}} k\right) .
$$

When the constant $c_{\beta_{n}}$ is large enough this proves our claim.

But the above statement follows directly at low enough temperatures, once we notice that

1) The function $\tilde{f}_{\beta}(n)$ is convex in $n$.

and

2) The differences $\left|\tilde{f}_{\beta_{n}}\left(n_{\max }\right)-\tilde{f}_{\beta_{n}}\left(n_{\max } \pm 1\right)\right|$ diverge when the sequence of inverse temperatures $\beta_{n}$ diverges; in fact these differences diverge proportionally to $3^{n}$.

\section{FINAL REMARKS AND CONCLUSION}

We have constructed a bounded-continuous-spin model with a bounded interaction, and a sequence of temperatures converging to zero, such that the (any) sequence of Gibbs measures at these temperatures does not converge to a ground state. This seems to be the first example in which a form of Chaotic Temperature Dependence has been proven.

In our example there are many more ground states than the ferromagnetic and antiferromagnetic ones, however, by adding non-nearest neighbor terms, one can suppress these if one wants.

Whether the phenomenon can also occur at positive temperatures, or for discrete spins, at this point remain open questions. However, it is known that for one-dimensional, sufficiently short-range, discrete-(finite-)spin interactions the Gibbs measures do converge to a limit - ground state - measure as the temperature decreases. $^{(2,5)}$

\section{ACKNOWLEDGMENTS}

We thank Christof Külske for some helpful remarks on the manuscript, and professor A.N. Berker for helpful correspondence on the literature. 


\section{REFERENCES}

1. M. Aizenman, J. T. Chayes, L. Chayes and C. M. Newman, Discontinuity of the magnetization in one-dimensional $\frac{1}{(x-y)^{2}}$ Ising and Potts models. J. Stat. Phys. 50:1-40 (1988).

2. J. Brémont, Gibbs measures at temperature zero. Nonlinearity 16:419-426 (2003).

3. A. C. D. van Enter and S. B. Shlosman, Provable first-order transitions for nonlinear vector and gauge models with continuous symmetry. Comm. Math. Phys. 255:21-32 (2005).

4. H.-O. Georgii, Gibbs measures and phase transitions. De Gruyter, Berlin (1988).

5. R. Leplaideur, A dynamical proof for the convergence of Gibbs measures at temperature zero. Nonlinearity 18:2847-2880 (2005).

6. S. R. McKay, A. N. Berker and S. Kirkpatrick, Spin-Glass Behavior in Frustrated Ising Models with Chaotic Renormalization-Group Trajectories. Phys. Rev. Lett. 48:767-770 (1982).

7. C. M. Newman and D. L. Stein, Ordering and broken symmetry in short-range spin glasses (Topical Review). J. Phys. Condens. Matter 15:R1319-1364 (2003).

8. C. M. Newman and D. L. Stein, Short-range spin glasses: results and speculations. To appear in the Monte Verita proceedings on Spin Glasses. arXiv cond-mat/0503345, Springer Lecture Notes in Mathematics 1900 (2006).

9. E. A. Pecherski and S. B. Shlosman, Low-temperature phase transitions in systems with one ground state. Theor. Math. Phys. 70:325-330 (1987).

10. D. Ruelle, Some ill-defined problems on regular and messy problems in statistical mechanics and smooth dynamics for which I would like the advice of Yasha Sinai. J. Stat. Phys. 108:723-728 (2002).

11. D. J. Thouless, The long-range order in one-dimensional Ising systems. Phys. Rev. 187:732-733 (1969).

12. Dr Seuss, The Cat in the Hat comes back. New York, Random House (1958). 